Wirtschaftskybernetik und Systemanalyse

Herausgegeben von den Professoren

Thomas Fischer und Meike Tilebein

Band 29

\title{
Modellbasiertes Management
}

Konferenz für Wirtschafts- und Sozialkybernetik KyWi 2013 vom 4. bis 5. Juli 2013 in Bern

\section{Herausgegeben von}

Stefan N. Grösser, Markus Schwaninger, Meike Tilebein, Thomas Fischer und Sabina Jeschke

Tab., Abb.; 399 S. 2014

Print: $\langle 978-3-428-14267-5\rangle € 99,90$

E-Book: $\langle 978-3-428-54267-3\rangle € 89,90$

Print \& E-Book: $\langle 978-3-428-84267-4\rangle € 119,90$

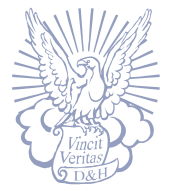

Duncker \& Humblot • Berlin 
Modellbasiertes Management (MBM), das Hauptthema dieses Bandes, ist ein Forschungskonzept, dessen Ziel es ist, das Management komplexer Situationen und Systeme zu verbessern. Konkret sollen Modelle und Methoden für eine verbesserte Entscheidungsfindung bereitgestellt sowie deren Effektivität im natürlichen Kontext getestet werden.

Die Grundlage des modellbasierten Managements bildet das Conant Ashby Theorem, welches besagt, dass die Effektivität eines Management-Prozesses nicht besser als das Modell sein kann, auf dem der Prozess aufbaut. Dieses Gesetz hat universelle Gültigkeit, da wir Entscheidungen stets auf der Grundlage von Modellen treffen - ob wir das wollen oder nicht und ob wir es wissen oder nicht.

Im Lichte des Conant-Ashby-Theorems sind gute Modelle die vielleicht wichtigste Voraussetzung für organisatorische (Über-) Lebensfähigkeit. Damit ist das Streben nach hochwertigen Modellen zwingend.

\section{Inhaltsverzeichnis}

\section{Einführung in Modellbasiertes Management}

Markus Schwaninger und Stefan N. Grösser: Kybernetische Grundlagen eines modellbasierten Managements $\ldots \ldots \ldots \ldots \ldots \ldots \ldots \ldots$

Falko E. P. Wilms: Unterscheidung als Grundoperation in sozialen Systemen - Eine sozialkybernetische Betrachtung ..............

II. Geschäftsmodellanalyse

Stefan N. Grösser und Michael Bürgi: Analyse von Geschäftsmodellen und Entwicklung von Maßnahmen durch computerbasierte

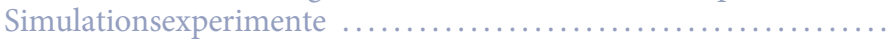

Sebastian Köpp, Aliaksei Mukhachou and Markus Schwaninger: Fixed-offer Group Buying Schemes - A Sustainable Business Model? ..............................................

Christian Tummel, Eckart Hauck und Sabina Jeschke: Exklusive Frachtlinien auf Basis eines elliptischen Mehrzonenmodells zur formalen Beschreibung des CloudLogistic-Konzepts .............

Phil Friedrichsmeier, Katharina Tarnacki, Eckart Hauck, Frank Hees und Sabina Jeschke: Entwicklung einer Business-Architektur für eine Frachtenkooperation kleiner und mittlerer Speditionsunternehmen im Teilladungssegment $\ldots \ldots \ldots \ldots \ldots \ldots \ldots \ldots \ldots \ldots$.

Maximilian Happach und Meike Tilebein: Ein Modell zur Einführung des Einwegpfands in Deutschland.$\ldots \ldots \ldots \ldots \ldots \ldots \ldots \ldots \ldots \ldots$ 


\section{Modellbasierte Strategie, Planung und Umsetzung}

Robert Rieg: Dynamik immaterieller Werte und Unternehmenserfolg: Folgerungen für die Messung des Zusammenhangs .........

Markus Schwenke und Stefan N. Grösser: Modellbasiertes Management für dynamische Problemstellungen zur Erweiterung statischer Managementwerkzeuge .........................

Stefan Kermer: Strategische Planung durch Modellierung aus Sicht eines Energieversorgungsunternehmens am Wärmemarkt

Margret Richter: Erneuerung von Strategieplanungsprozessen - biokybernetisch überprüft ..............................

Philipp Wolters: Entwicklung einer kybernetisch motivierten $\mathrm{Me}$ thodik zur Ermittlung des Wertbeitrags der Unternehmens-IT ....

Andreas Ninck und Beat Estermann: Systemische Wirkungsanalyse als Grundlage für die strategische Planung einer Online-Community

Martin F. G. Schaffernicht: Über die komplexe Aufgabe, betriebliche Ressourcen zu steuern: der Governator als modellgestützte

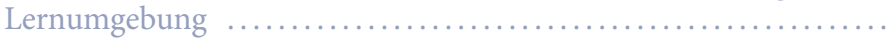

\section{Generic Governance}

Ralf-Eckhard Türke: Generic Governance ...................

Heiko Eckert: Generic Governance II - Systemische Strategieent-

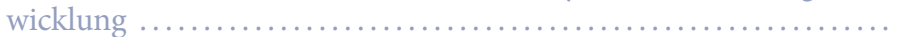

Remo Berlanda: Mit Kybernetik zu nachhaltiger Entwicklung - Das

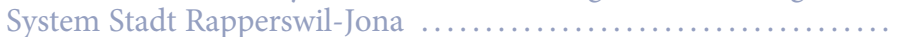

V. Methoden für Innovations- und Personalmanagement

Philipp Wunderlich, Nicole Zimmermann und Andreas Größler: Management der Diffusion von Innovationen in intra-organisationalen Netzwerken unter Verwendung von System Dynamics .....

Andreas M. Krafft: Innovationskraft und Wohlbefinden im Management: Modellbildung und empirische Evidenz ................

Nicole Zimmermann: Modellbasierte Analyse des Managements monetärer Anreize bei reziproken und opportunistischen Mitarbeiterschaften

Yumino Endres, Ulrike Maier and Axel Löffler: A System Dynamics Model for the Admission Management of Study Programs ....

\section{Anwendungen von Modellbasiertem Management}

Thorsten Theisinger und Fabio Squillante: Das agenten-basierte Dynamische Marktmodell der Deutschen Telekom AG als Beispiel

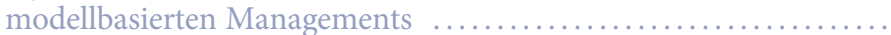

Werner Boysen: BoardRoom - ein neuer Ansatz zur Unterstützung komplexer Entscheidungsprozesse $\ldots \ldots \ldots \ldots \ldots \ldots \ldots \ldots \ldots$

Berend Faber-Praetorius: Modelle komplexitätsgerechten Projektmanagements im Lebenszyklus der Immobilie ............... 


\section{Bestellung}

Hiermit bestelle ich:

...... Expl. Modellbasiertes

Management

Konferenz für Wirtschafts- und Sozialkybernetik KyWi 2013 vom 4. bis 5. Juli 2013 in Bern

Herausgegeben von Stefan N. Grösser, Markus Schwaninger, Meike Tilebein, Thomas Fischer und Sabina Jeschke

Wirtschaftskybernetik und Systemanalyse, Band 29 Tab., Abb.; 399 S. 2014

$\square$ Print: $\langle 978-3-428-14267-5\rangle € 99,90$

$\square$ E-Book: $\langle 978-3-428-54267-3\rangle € 89,90$

$\square$ Print \& E-Book: $\langle 978-3-428-84267-4\rangle € 119,90$

Absender:

E-Mail:

Datum, Unterschrift:

Bestellungen können an jede Buchhandlung oder direkt an den Verlag gerichtet werden. Nutzung und Download von E-Books erfolgen über unsere eLibrary. Privatkunden erhalten den Zugang direkt beim Erwerb des E-Books über unseren Web-Shop.

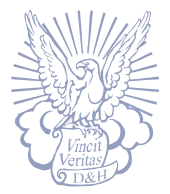

\section{Duncker \& Humblot GmbH • Berlin}

Postfach 410329 . D-12113 Berlin · Telefax (0 30) 79000631 www.duncker-humblot.de 